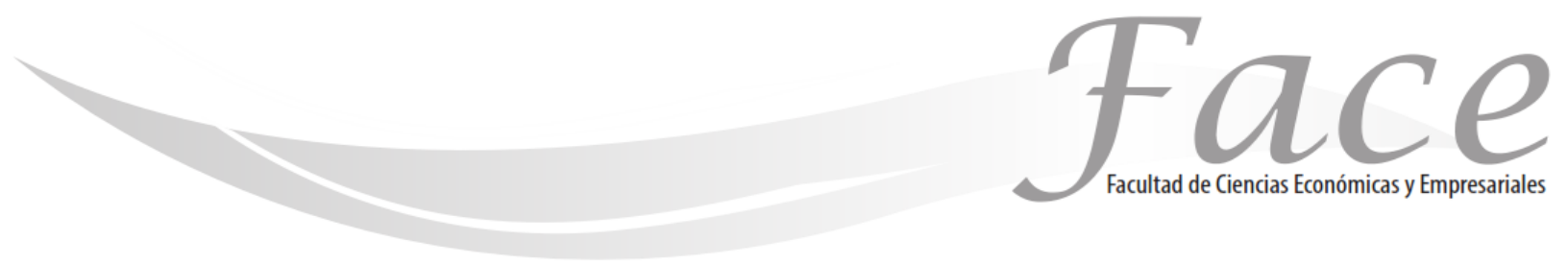

ISSN Impreso: 1794-9920

ISSN Electrónico: 2500-9338

Volumen $18-\mathrm{N}^{\circ} 1$

Año 2018

Págs. 44 - 52

\title{
MODELOS MEDIA MÓVIL SIMPLE Y MEDIA MÓVIL EXPONENCIAL COMO PRONÓSTICO DE \\ LA ACCIÓN DE ISA
}

\author{
Jorge Enrique Díaz Pinzón * \\ Enlace ORCID: https://orcid.org/0000-0002-8870-7769
}

Fecha de Recepción: 27 de abril 2018

Fecha de Aprobación: 17 de Julio 2018

\section{Resumen:}

Se realizó un estudio del análisis, y modelo de predicción, se efectuó sobre las acciones de ISA, durante el período enero-marzo de 2018. El objetivo del trabajo de investigación fue realizar una predicción econométrica de media móvil simple y media móvil exponencial de la acción de ISA durante el trimestre enero-marzo de 2018. Se ejecutó un modelo predictivo econométrico media móvil simple y media móvil exponencial, mediante el paquete Excel, para predecir la dirección del precio de la acción. Se pudo establecer que el precio de la acción de ISA en el trimestres en estudio se sitúo en los $\$ 13320$, y un pronóstico de $\$ 13160$, lo que representa una disminución de alza de la acción, comparada con el inicio del trimestre en enero de 2018 que fue de \$ 14140.

Palabras Clave: Acciones; Análisis estadístico; Indicador económico; Media móvil.

*Magíster en Gestión de la Tecnología Educativa. UDES. Docente de matemáticas e investigador. Correo: jediazp@unal.edu.co. 
MÍDIA MÓVEL SIMPLES

\title{
MÍDIA MÓVEL SIMPLE AND MOBILE EXPONENTIAL MEDIA AS A PROGNOSIS OF \\ THE ACTION OF ISA
}

\begin{abstract}
:
A study of the analysis, and prediction model, was carried out on the actions of ISA, during the period January-March 2018. The objective of the research work was to perform an econometric prediction of simple moving average and exponential moving average of the share of ISA during the January-March quarter of 2018. A simple mobile average and exponential moving average econometric predictive model was executed, using the Excel package, to predict the direction of the share price. It was established that the price of the ISA share in the quarters under study stood at $\$ 13320$, and a forecast of $\$ 13160$, which represents a decrease in the share's rise, compared to the start of the quarter in January. 2018 that was $\$ 14140$.
\end{abstract}

Keywords: Actions; Statistic analysis; Economic indicator; SMA.

MODELOS MÓVEIS MÓVEIS E MÓVEIS EXPONENCIAIS MÓVEIS COMO UM PROGNÓSTICO

A AÇÃO DO ISA

\section{Resumo:}

Um estudo da análise e do modelo de predição foi realizado sobre as ações do ISA, no período de janeiro a março de 2018. 0 objetivo do trabalho de pesquisa foi realizar uma previsão econométrica da média móvel simples e exponencial da média móvel do trabalho. parte da ISA durante o trimestre janeiro-março de 2018. Um modelo preditivo econométrico médio móvel simples e exponencial móvel médio foi executado, usando o pacote Excel, para prever a direção do preço da ação. Foi estabelecido que o preço da ação da ISA nos trimestres em estudo era de US $\$ 13320$, e uma previsão de US $\$ 13.160$, o que representa uma queda no aumento da ação, em comparação com o início do trimestre em janeiro. 2018 que foi de US $\$ 14140$.

Palavras chave: Ações; Análise estatística; Indicador econômico; SMA. 


\section{INTRODUCCIÓN:}

En los mercados bursátiles según (BBVA, s.f), la mayoría de las cotizaciones de las acciones se mueven por tendencias a corto plazo. Asimismo pueden rendir unos resultados muy buenos y la notoriedad que hacen que aumente su valor. Sin embargo, hay que tener atención ya que estas tendencias alcanzar cualquier variación inesperada en cualquier momento y por lo tanto la previsión de futuros movimiento será equívoca.

Ahora definamos la pregunta problema: ¿Es posible predecir el precio de la acción de ISA a través del análisis de series históricas de precios?

Según lo anterior, surge el interrogante acerca de qué tan eficaces son los pronósticos de precios en los activos financieros apoyados en el análisis estadístico de series de tiempo. Al respecto, si fuese factible esbozar un modelo de pronóstico fundamentado en la serie previamente de los precios, sería viable entonces obtener beneficios económicos prematuramente de los precios futuros e incluso, que éstos sean muy por encima de los habitualmente promete el mercado. (García 2010).

La Interconexión Eléctrica S.A. E.S.P. - ISA - es una Compañía multilatina con representación en 8 países y despliega sus actividades a través de 33 empresas, en 4 líneas de negocios que le han concedido fortalecerse durante más 49 años: Transporte de Energía Eléctrica, Concesiones Viales, Tecnologías de Información y Telecomunicaciones y Gestión de Sistemas de Tiempo Real. (ISA, 2018).

La composición accionaria de la empresa ISA, es una "Empresa de Servicios Públicos Mixta con inversionistas estatales, públicos y privados. Las acciones en circulación son acciones ordinarias, nominativas y desmaterializadas. El Depósito Centralizado de Valores de Colombia DECEVAL S.A.-, es la entidad que recibe en depósito los valores para su administración y custodia contribuyendo a facilitar y agilizar las operaciones de los agentes del mercado". (ISA, 2018). El Capital suscrito y pagado en circulación es de 1.107.677.894 acciones.

Según (Arboleda, 2004), las acciones nominativas son las que se consignan a nombre de una persona explícita inscribiéndola en el libro de registro de accionistas de la sociedad que las prorrumpe.

Según (Economipedia, 2018), Una acción ordinaria es una acción que se puede comercializar en el mercado y que incorpora una parte conveniente del capital social de una empresa. Este título o valor financiero da derecho a su poseedor a ser dueño de la empresa en la parte que le pertenezca.

La emisión desmaterializada es aquella que no pretende la expedición de títulos físicos individuales para proteger cada colocación. Entre el emisor y DECEVAL se firma un contrato de depósito de emisiones y se desembolsa un macro título que cubre una parte del conjunto de la emisión. (DECEVAL, 2018).

El objetivo del trabajo de investigación fue realizar una predicción econométrica de media móvil simple y media móvil exponencial de la acción de ISA durante el trimestre enero-marzo de 2018, con una base de sesenta datos.

\section{MARCO REFERENCIAL:}

Los estudios sobre pronósticos de valores futuros de activos financieros, adquiriendo como insumo el comportamiento pasado de estos, han venido utilizando herramientas estadísticas con las que han obtenido resultados muy ventajosos en la mayoría de los casos. Al respecto Lamberton (1996) citado por (Parody, 2016) confirma que el volumen de negociación de estos activos financieros es tan significativo, que se han desarrollado variados modelos matemáticos con el fin de poder pronosticar el valor que puede tomar una acción en el futuro.

Según ( Parody,2016), el comportamiento de las acciones en Colombia ha sido desarrollado con diferentes métodos y desde diferentes grupos de acciones; Cruz, Medina y Zapata (2010) analizan la tendencia de los valores del IGBC durante el 2009, en el cual esgrimen el método de caminata aleatoria, estableciendo que el índice tiene una contrastada tendencia al alza y su componente aleatorio 0 ruido blanco es poco revelador visto en su representación gráfica y que en general los precios de las acciones de la Bolsa de Valores de Colombia no persiguen la caminata aleatoria.

Media móvil simple (SMA del inglés): "Mediante el enlace "Med. Móvil" en los gráficos, verá la media móvil de la cotización que se consigue sumando los precios medios del valor para los periodos bajo estudio ("n") y, después, dividiendo el total obtenido por esa misma cantidad de periodos ("n")". (Domínguez, 2008)

Según (Mateu, 2018). Las medias móviles son indicadores que aplanan 0 pulen, en mayor 0 menor medida, el progreso de los precios, de tal forma que 
eliminan determinadas oscilaciones, sean a corto, medio o largo plazo.

Media móvil exponencial (EMA del inglés): "La diferencia entre la media móvil exponencial y la media móvil simple es que la exponencial da más peso a los datos más recientes. De esta manera, los inversores podrán hacer un mejor seguimiento del valor y responder rápidamente a las tendencias que pueden tardar más tiempo en aparecer en un gráfico de la media móvil simple." (Domínguez, 2008)

\section{METODOLOGÍA:}

Para conseguir el objetivo propuesto en esta investigación, se utilizó la base de datos del precio de cierre de la acción de ISA en la bolsa de Valores de Colombia (BVC, 2018), durante el período comprendido entre enero y marzo de 2018, con un total de datos de sesenta. El tipo de media móvil a utilizar fue: medias móviles (simple y exponencial) a partir de los precios de cierre, para su construcción se toman únicamente los precios de cierre de la acción de ISA diariamente.

Según (Trucos y cursos, 2015), el análisis de la media móvil programa valores en el período de predicciones, fundamentado en el valor promedio de la variable calculada durante un número delimitado de periodos anteriores.

La media móvil nos suministra información de predilecciones que se vería oculta por una simple media de todos los datos históricos.

El autor (Sáenz, 2016), menciona que sin duda alguna, la media móvil más usada por los traders (comerciantes) es la media móvil simple, por su sencillez de cálculo y que es uno de los indicadores de sobresalientes señales de compra y venta contribuye, siendo éstas claras y concisas. La fórmula de la media móvil simple es la siguiente:

Media Móvil Simple $=\frac{\text { Suma Precio Cierre de X sesiones }}{X}$

\section{RESULTADOS:}

En la tabla 1 (Ver Anexo 1), se observan el precio de cierre de la acción de ISA durante el primer trimestre del año 2018 y utilizando la formula (1), la media móvil simple con intervalo 3 , los primeros datos con N/A, se da porque para calcular la SMM no tienen dos datos anteriores al dato central, de ahí en adelante se toma el dato central y los dos datos anteriores, se suman y se dividen entre tres.

En la tabla 2 (Ver Anexo 2), se observan el precio de cierre de la acción de ISA durante el primer trimestre del año 2018 y utilizando la formula (2), la media móvil exponencial con intervalo 3 , los primeros datos con N/A, se da porque para calcular la EMA no tienen dos datos anteriores al dato central, Para calcular la media móvil exponencial se utilizó la fórmula:

$E M A=$ el precio actual * $K+$ EMA de ayer * $(1-K)$ cuando $\mathrm{K}=2 /(\mathrm{N}+1)$

Figura 1. Representación gráfica de la progreso de la cotización de las acciones de ISA (02/01/2018-29/03/2018). Media móvil (SMM) (60 días)

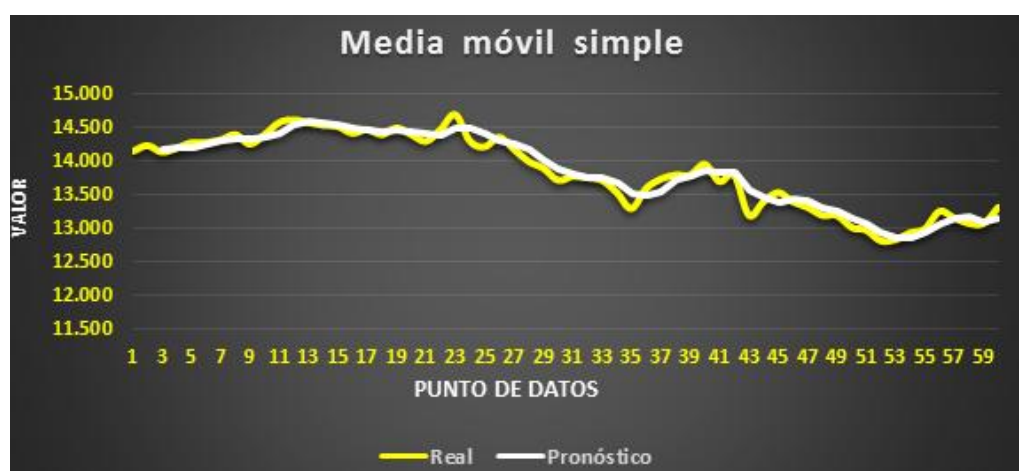

Fuente: Elaboración propia

En la figura 1, representa en amarillo el precio real de la acción de ISA en la Bolsa de Valores de Colombia en el intervalo enero-marzo de 2018. Se puede apreciar, la eliminación de la volatilidad mediante la media móvil. También, el cambio de tendencia producido, cuando la cotización de la acción penetra la media móvil, y podemos ver como se transforma en algo análogo a lo que podríamos nombrar "resistencia" cuando baja el precio de cierre de la acción, y la media móvil pasa a ubicarse por encima del valor actual. Siguiendo esta misma teoría, 
ISSN: 1794-9920 - ISSN Electrónico: 2500-9338

Enero - Julio de 2018

Volumen 18 Número 1, Año 2018 Págs. 44 - 52

cuando el precio de la cotización aumenta y sobresale la media móvil, ésta pasa a formar un nivel de "soporte".

También podemos analizar que el precio al final de la acción en el trimestres en estudio se sitúo en los \$13320, y un pronóstico de $\$ 13160$, lo que representa un baja en la cotización de la acción, comparada con el inicio del trimestre en enero de 2018 , que fue de $\$ 14140$.

Se presenta también varias oscilaciones en el precio de la acción debido a la volatilidad de la misma. Los picos más bajos se presentan en los días 35,43 y 53 del trimestre, los picos más altos se presentan en los días 12 y 23 del trimestre en estudio.

La media móvil en el grafico ha producido varios cortes el precio de cotización de la acción, esto quiere decir que la MMS se ajusta bastante bien a los datos reales. El corte de los precios sobre la media móvil puede sugerirnos de que se ha producido un cambio de tendencia.

Según (Matéu, 2018) "Mientras los precios o cotizaciones se muevan o se encuentren por encima de la media móvil el valor o mercado estará en tendencia alcista y, por tanto, se debe estar "comprado" hasta el momento en que se produzca la señal de venta".

Cuando los precios o cotizaciones de la acción se desplacen o se encuentren por debajo de la media móvil el valor o mercado quedará en tendencia bajista y, por tanto, se debe estar "vendiendo" o evitar tomar posiciones en ellos. (Mateu, 2018)

Figura 2. Representación gráfica de la progreso de la cotización de las acciones de ISA (02/01/2018-29/03/2018). Media móvil exponencial (EMA) (60 días)

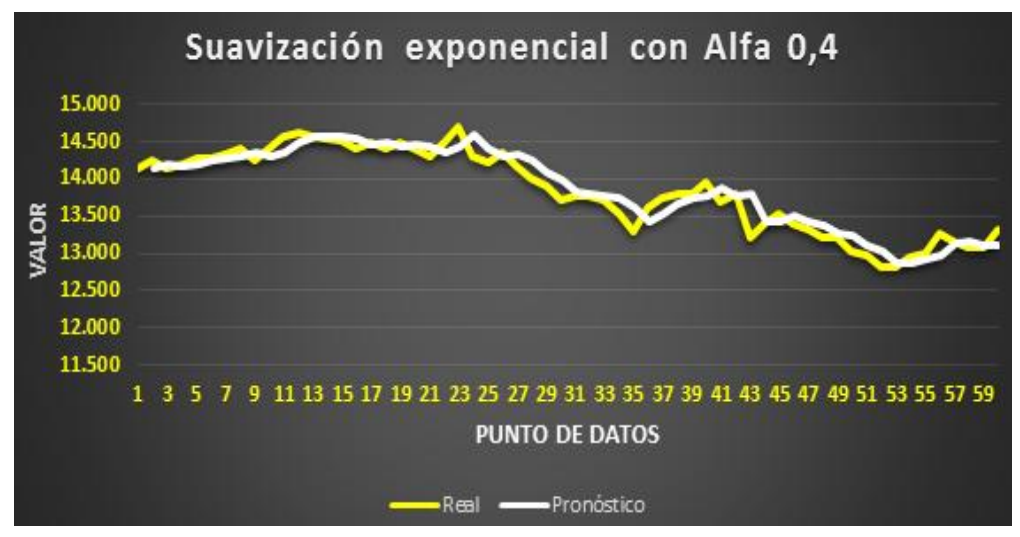

Fuente: Elaboración propia mediante Excel
En la figura 2, se aprecia en amarillo el precio real de la acción de ISA en la Bolsa de Valores de Colombia en el intervalo enero-abril de 2018. Se puede apreciar, la eliminación de la volatilidad mediante la media móvil exponencial. También, el cambio de tendencia producido, cuando la cotización de la acción penetra la media móvil exponencial, y podemos ver como se transforma en algo análogo a lo que podríamos nombrar "resistencia" cuando baja el precio de cierre de la acción, y la media móvil pasa a ubicarse por encima del valor actual. Siguiendo esta misma teoría, cuando el precio de la cotización aumenta y sobresale la media móvil, ésta pasa a formar un nivel de "soporte".

Según (Efxto, 2018), La media móvil exponencial, al igual que la media móvil simple, brinda una correlación afinada entre la acción del precio y el lapso del tiempo. La diferencia está en que el cálculo de la media móvil exponencial da más categoría a los últimos datos alcanzados durante un explícito período.

"La fórmula de media móvil exponencial es una media móvil de datos que da más peso a los datos más recientes del período y menos peso a los datos más antiguos del período". (MSDN, 2018).

\section{DISCUSIONES:}

Según (Ruiz, et al. 2004), la utilización de indicadores propuestos por el análisis técnico, planteando operaciones de compra y de venta de un instrumento financiero únicamente en función de las señales que las medias móviles favorecen y transmiten automáticamente el oportuno orden del mercado. Se han identificado una serie de supuestos en los que la aplicación por determinados agentes de una estrategia basada en el cruce de medias móviles, se pueden lograr rendimientos significativos.

Asimismo se pone de manera palpable que las estrategias con medias móviles simples son las que tienen un sobresaliente comportamiento en los diferentes escenarios de costos de transacción. (Miralles, Miralles, 2005).

A corto plazo los resultados que proporcionan la utilización de medias móviles están condicionados por la tendencia del mercado del momento. No se puede augurar, que un inversor que utilice aquellas medias móviles que mejor se hayan comportado en el pasado vaya obtener un beneficio extraordinario en el corto plazo. (Miralles, Miralles, 2005).

Se puede apreciar, la eliminación de la volatilidad mediante la media móvil exponencial. También, el cambio de tendencia producido con el mercado accionario. (Díaz, 2018). 


\section{CONCLUSIONES:}

En el presente trabajo de investigación se pudo evidenciar la predictibilidad en el precio de la acción de ISA a través de la media móvil simple y suavización exponencial. Se determinó que el precio al final de la acción en el trimestres en estudio se sitúo en los \$13320, y un pronóstico de \$ 13160 , lo que representa un baja en la cotización de la acción, comparada con el inicio del trimestre en enero de 2018, que fue de $\$ 14140$.

Es posible elaborar modelos de pronóstico de activos financieros bajo el modelo de media móvil simple y media móvil exponencial esgrimiendo parámetros estadísticos para su evaluación, realizando simulaciones para los valores pronosticados a partir de los valores de series periódicas, en este caso series trimestrales de las acciones de ISA esencia del estudio.

Esto lo recomienda (Parody, et al, 2016), que la aplicación de modelos con promedios móviles de suavizamiento exponencial y modelos de la familia Arch y Garch generan mayor capacidad de predicción dado que permiten mejor modelamiento de su volatilidad.

Se observó una baja en la cotización de la acción de ISA en la BVC, en el período estudiado, esto implica que los tenedores de las acciones no puedan vender a un buen precio sus títulos valores.

En consonancia con (Parody, et al, 2016), empleando las simulaciones, no alcanza a dilucidar todos los eventos que se originan en el mercado, por lo que a los activos más aéreos, se les crea menor capacidad predictiva; en este orden de ideas el modelo demostró mayor exactitud en la predicción del precio de la acción de ISA.

Alguna de las limitantes del trabajo como lo menciona (Pérez, et al, 2012), está en divulgar un método de pronósticos por categoría ocasiona alejamiento de éste con la demanda real, en la mayoría de los casos. La elección del conspicuo método está dada por divergencias muy pequeñas entre sus errores; en ocasiones, al reconstruir la información, la elección del método varía con respecto a un espacio de tiempo pasado.

\section{REFERENCIAS:}

Arboleda, L (2004). Las acciones de la sociedad anónima. Recuperado el 28 de abril de 2018 de: http://www.javeriana.edu.co/biblos/tesis/derecho/ dere3/tesis08.pdf

Banco Bilbao Vizcaya Argentaria. BBVA. (s.f). Factores que influyen en el precio de las acciones. Recuperado el 18 de septiembre de 2018 de: https://www.bbva.es/estaticos/mult/Ayudas_factor es_acciones.pdf_tcm924-528182.pdf

Bolsa de Valores de Colombia. BVC. (2018). Mercados en línea. Recuperado el 27 de abril de 2018 de: http://www.bvc.com.co/pps/tibco/portalbvc/Home/ Mercados/enlinea/acciones\#

Deposito Descentralizado de Valores de Colombia. DECEVAL (2018). Recuperado el 28 de abril de 2018 de: https://www.deceval.com.co/portal/page/portal/Ho me/Servicios/Emisiones_Desmaterializadas

Díaz, J. (2018). Análisis Econométrico de la acción de Ecopetrol. Editorial Académica Española. ISSN: 978-620-2-16002-5.

Domínguez, J. (2008). Como se calcula la media móvil simple. Recuperado el 24 de abril de 2008 de: https://juandominguez.wordpress.com/2008/06/22 /los-indicadores-de-tendencia/

ECONOMIPEDIA. (2018). Acción ordinaria. Recuperado el 24 de abril de 2018 de: http://economipedia.com/?s=accion+ordinaria

Efxto (2018). Media móvil exponencial. Recuperado el 28 de abril de 2018 de: https://efxto.com/escuela/media-movilexponencial

García, J. (2010). Predictibilidad en los retornos de la acción de Ecopetrol: Evidencia a través del efecto día. Recuperado el 18 de septiembre de 2018 de: https://repository.upb.edu.co/bitstream/handle/20. 500.11912/983/digital_19822.pdf 

de 2018 de: http://www.isa.co/es/relacion-conformulas-ejemplos inversionistas/Paginas/perfil-de-la-compania.aspx

Matéu, J. (2018). Diccionario económico. Recuperado el 24 de abril de 2018 de: http://www.expansion.com/diccionarioeconomico/media-movil.html

Miralles, J. Miralles, M. (2005). Estabilidad de las estrategias de inversión con medias móviles. Revista Española de Financiación y Contabilidad. Vol. XXXIV, No. 127. Octubre-diciembre 2005. Pp 849-873. Recuperado el 24 de abril de 2018 de: https://dialnet.unirioja.es/descarga/articulo/14019 81.pdf

MSDN (2018). Media móvil exponencial. Recuperado el 24 de abril de 2018 de: https://msdn.microsoft.com/eses/library/dd456643.aspx

Parody, E. Charris, A. Garcia. R. (2016). Modelo log-normal para predicción del precio de las acciones del sector bancario. Recuperado el 24 de abril de 2018 de: http://www.scielo.org.co/pdf/diem/v14n1/v14n1a1 0.pdf

Pérez, R. Mosquera, S. Bravo, J. (2012). Aplicación de modelos de pronósticos en productos de consumo masivo. Recuperado el 18 de septiembre de 2018 de: http://www.scielo.org.co/pdf/bsaa/v10n2/v10n2a1 4.pdf

Trucos y Cursos. (2018).El análisis de la Media móvil en Excel proyecta valores en el período de pronósticos, basándose en el valor promedio de la variable calculada durante un número específico de periodos anteriores. Recuperado el 24 de abril de 2018 de: http://trucosycursos.es/lamedia-movil-en-excel/

Ruiz, V. Pérez, M. Olasolo, A. (2004). Análisis de la eficacia de las medias móviles en el mercado intradiario de renta variable español. Recuperado el 08 de septiembre de 2018 de: https://dialnet.unirioja.es/descarga/articulo/27477 43.pdf

Sáenz, F. (2016). Medias móvil simple, exponencial y ponderada: formulas y ejemplos. Recuperado el 24 de abril de 2018 de: https://www.rankia.cl/blog/analisis-ipsa/2039072- 


\section{ANEXO 1}

Tabla 1. Media móvil (SMM) de la acción de ISA

\begin{tabular}{|c|c|c|c|}
\hline Día & Precio de cierre & Media Móvil & Error Típico \\
\hline 1 & 14.140 & & \\
2 & 14.240 & \#N/A & \#N/A \\
3 & 14.140 & \#N/A & \#N/A \\
4 & 14.200 & 14.173 & \#N/A \\
5 & 14.280 & 14.193 & \#N/A \\
6 & 14.280 & 14.207 & 46,66666667 \\
7 & 14.320 & 14.253 & 45,21553322 \\
8 & 14.400 & 14.293 & 47,60952286 \\
9 & 14.260 & 14.333 & 44,22166387 \\
10 & 14.400 & 14.327 & 56,56854249 \\
11 & 14.580 & 14.353 & 60,73622386 \\
12 & 14.620 & 14.413 & 107,0825227 \\
13 & 14.580 & 14.533 & 111,7536974 \\
14 & 14.540 & 14.593 & 108,7300429 \\
15 & 14.520 & 14.580 & 55,64437345 \\
16 & 14.420 & 14.547 & 28,80329199 \\
17 & 14.480 & 14.493 & 50,62571444 \\
18 & 14.400 & 14.473 & 45,21553322 \\
19 & 14.500 & 14.433 & 46,66666667 \\
20 & 14.400 & 14.460 & 30,30707044 \\
21 & 14.300 & 14.433 & 35,69417423 \\
22 & 14.480 & 14.400 & 65,09252674 \\
23 & 14.700 & 14.393 & 78,78710178 \\
24 & 14.300 & 14.493 & 141,6830056 \\
25 & 14.220 & 14.493 & 170,8800749 \\
26 & 14.360 & 14.407 & 195,7322434 \\
27 & 14.180 & 14.293 & 159,8610508 \\
28 & 14.000 & 14.253 & 122,0200348 \\
29 & 13.900 & 14.180 & 118,6342028 \\
30 & 13.720 & 14.027 & 133,9430504 \\
31 & 13.780 & 13.873 & 154,8715119 \\
32 & 13.760 & 13.800 & 115,405886 \\
33 & 13.720 & 13.753 & 89,35986339 \\
34 & 13.540 & 13.753 & 22,7710017 \\
35 & 13.300 & 13.673 & 79,44250192 \\
36 & 13.600 & 13.520 & 149,7652484 \\
37 & 13.740 & 13.480 & 163,8879473 \\
38 & 13.800 & 13.547 & 182,7364019 \\
39 & 13.800 & 13.713 & 140,5808058 \\
40 & 13.960 & 13.780 & 122,8669862 \\
41 & 13.700 & 13.853 & 80,18497135 \\
42 & 13.800 & 13.820 & 93,4126647 \\
43 & 13.200 & 13.820 & 93,4126647 \\
44 & 13.400 & 13.567 & 223,0429289 \\
45 & 13.540 & 13.467 & 215,4753574 \\
46 & 13.400 & 13.380 & 234,1572754 \\
47 & 13.320 & 13.447 & 103,637545 \\
48 & 13.200 & 13.420 & 112,2167215 \\
49 & 13.200 & 13.307 & 88,61067572 \\
50 & 13.020 & 13.240 & 87,51719408 \\
51 & 12.980 & 13.140 & 95,52971227 \\
52 & 12.820 & 13.067 & 88,52704128 \\
53 & 12.840 & 12.940 & 110,0168337 \\
54 & 12.940 & 12.880 & 88,52704128 \\
55 & 13.000 & 12.867 & 84,41519961 \\
56 & 13.260 & 12.927 & 64,17568479 \\
57 & 13.160 & 13.067 & 126,6666667 \\
58 & 13.080 & 13.140 & 119,9382557 \\
59 & 13.080 & 13.167 & 122,8669862 \\
60 & 13.320 & 13.107 & 53,61039147 \\
& 106 & 1061701420
\end{tabular}


ISSN: 1794-9920 - ISSN Electrónico: 2500-9338

Enero - Julio de 2018

Volumen 18 Número 1, Año 2018 Págs. 44 - 52

\section{ANEXO 2}

Tabla 2. Media móvil exponencial (EMA) de la acción de

ISA

\begin{tabular}{|c|c|c|c|}
\hline Día & Precio de cierre & Media Móvil & Error Típico \\
\hline 1 & 14.140 & & \\
\hline 2 & 14.240 & $\# N / A$ & $\# \mathrm{~N} / \mathrm{A}$ \\
\hline 3 & 14.140 & 14.140 & \#N/A \\
\hline 4 & 14.200 & 14200 & $\# \mathrm{~N} / \mathrm{A}$ \\
\hline 5 & 14.280 & 14164 & $\# N / A$ \\
\hline 6 & 14.280 & 14185,6 & 70,4651214 \\
\hline 7 & 14.320 & 14242,24 & 67,84138363 \\
\hline 8 & 14.400 & 14264,896 & 62,27138883 \\
\hline 9 & 14.260 & 14297,9584 & 66,76732838 \\
\hline 10 & 14.400 & 14359,18336 & 70,41485768 \\
\hline 11 & 14.580 & 14299,67334 & 88,10273898 \\
\hline 12 & 14.620 & 14359,86934 & 100,5240683 \\
\hline 13 & 14.580 & 14491,94774 & 150,9528462 \\
\hline 14 & 14.540 & 14568,77909 & 158,0298801 \\
\hline 15 & 14.520 & 14575,51164 & 147,1742729 \\
\hline 16 & 14.420 & 14554,20466 & 76,99430217 \\
\hline 17 & 14.480 & 14533,68186 & 29,1944262 \\
\hline 18 & 14.400 & 14465,47274 & 71,54159765 \\
\hline 19 & 14.500 & 14474,1891 & 69,0520705 \\
\hline 20 & 14.400 & 14429,67564 & 78,82180144 \\
\hline 21 & 14.300 & 14471,87026 & 59,61146176 \\
\hline 22 & 14.480 & 14428,7481 & 72,14538938 \\
\hline 23 & 14.700 & 14351,49924 & 94,31670621 \\
\hline 24 & 14.300 & 14428,5997 & 112,9215843 \\
\hline 25 & 14.220 & 14591,43988 & 188,6324855 \\
\hline 26 & 14.360 & 14416,57595 & 241,5973046 \\
\hline 27 & 14.180 & 14298,63038 & 256,4094592 \\
\hline 28 & 14.000 & 14335,45215 & 206,030368 \\
\hline 29 & 13.900 & 14242,18086 & 148,9672296 \\
\hline 30 & 13.720 & 14096,87234 & 169,8854234 \\
\hline 31 & 13.780 & 13978,74894 & 201,3087686 \\
\hline 32 & 13.760 & 13823,49958 & 234,0664451 \\
\hline 33 & 13.720 & 13797,39983 & 189,3866819 \\
\hline 34 & 13.540 & 13774,95993 & 153,0163097 \\
\hline 35 & 13.300 & 13741,98397 & 45,86776082 \\
\hline 36 & 13.600 & 13620,79359 & 122,7692506 \\
\hline 37 & 13.740 & 13428,31744 & 221,153526 \\
\hline 38 & 13.800 & 13531,32697 & 240,2644064 \\
\hline 39 & 13.800 & 13656,53079 & 242,1623845 \\
\hline 40 & 13.960 & 13742,61232 & 176,637811 \\
\hline 41 & 13.700 & 13777,04493 & 149,9124103 \\
\hline 42 & 13.800 & 13886,81797 & 138,2622141 \\
\hline 43 & 13.200 & 13774,72719 & 154,5605161 \\
\hline 44 & 13.400 & 13789,89088 & 151,6709466 \\
\hline 45 & 13.540 & 13435,95635 & 357,5429643 \\
\hline 46 & 13.400 & 13414,38254 & 341,5176027 \\
\hline 47 & 13.320 & 13489,75302 & 348,828444 \\
\hline 48 & 13.200 & 13435,90121 & 91,52087056 \\
\hline 49 & 13.200 & 13366,36048 & 111,4576747 \\
\hline 50 & 13.020 & 13266,54419 & 128,0162798 \\
\hline 51 & 12.980 & 13226,61768 & 123,2031242 \\
\hline 52 & 12.820 & 13102,64707 & 157,8974396 \\
\hline 53 & 12.840 & 13029,05883 & 143,9459368 \\
\hline 54 & 12.940 & 12903,62353 & 183,8828996 \\
\hline 55 & 13.000 & 12865,44941 & 144,6787838 \\
\hline 56 & 13.260 & 12910,17977 & 133,3058358 \\
\hline 57 & 13.160 & 12964,07191 & 76,75375874 \\
\hline 58 & 13.080 & 13141,62876 & 183,6653674 \\
\hline 59 & 13.080 & 13152,6515 & 178,8655117 \\
\hline \multirow[t]{2}{*}{60} & 13.320 & 13109,0606 & 176,2471563 \\
\hline & & 13091,62424 & 46,40496429 \\
\hline
\end{tabular}

